\title{
Paesaggio naturale e politiche di sviluppo territoriale in Islanda'
}

\author{
by Emanuela Finocchietti - Luca Zarrilli
}

1. Introduzione - "In complesso è un cantuccio del mondo che risulta assai interessante. Come accade in parecchie altre piccole comunità, anche l'Islanda dimostra quanto possa l'ingegno umano contro le condizioni sfavorevoli della natura. Poiché l'Islanda non è certo un paradiso terrestre; se l'inverno non vi è eccessivamente freddo, grazie alla prossimità di una diramazione della Corrente del Golfo, d'altra parte l'estate vi è assai troppo breve per consentire la coltivazione sia dei cereali sia delle frutta. E inoltre piove incessantemente! [...] Nonostante queste condizioni scarsamente allettanti, v'è chi ci vive lassù, non solo, ma che per giunta ci si trova bene. Negli ultimi sessanta anni, circa ventimila Islandesi emigrarono in America, per lo più nel Manitoba. Ma molti si sono affrettati a tornare a casa. Piove. Non vi sono comodità. Ma si sentono a casa loro.” (Van Loon, 1949, pp. 171-172). Così, sul finire degli anni Trenta, nella sua curiosa Geografia, Hendrik Willem Van Loon descriveva l'Islanda, non immaginando che un giorno - peraltro non troppo lontano - in quell'interessante ma piovoso "cantuccio del mondo" si sarebbero coltivate banane! Sia pur in serre riscaldate con acqua calda sgorgata direttamente dalle viscere della Terra e veicolata attraverso un avveniristico sistema di pompaggio e di condotte. Era però ben consapevole di quanto forte fosse l'attaccamento del popolo islandese alla propria terra e quanto profondo il radicamento nel proprio territorio. Gli islandesi sanno di vivere in un contesto ambientale unico, nel bene e nel male, e ne sono fieri. Sono pochi gli islandesi che lasciano per sempre il proprio paese. "Linguaggio e memoria sono ciò che costituisce l'identità. Ma l'identità è fatta anche da una terra in movimento, dal suolo che si calpesta, dal rispetto che gli si porta. Dall'essere, come si dice, in un posto speciale nel mondo dove i continenti si sono distaccati. Una specie di luogo unico dove la gente è isolata, forse, ma vive in pace." (Viganò, 2004, p. 122). E' vero, gli islandesi vivono in pace con gli altri uomini (se si escludono le cod wars antibritanniche degli anni Sessanta e Settanta, poco più che incruente schermaglie navali), ma in costante "tensione" con la natura, foriera al tempo stesso di prosperità e di catastrofe. Come scrisse Giorgio Manganelli, approdato in Islanda negli anni Settanta in qualità di inviato del quotidiano "La Stampa": "Questa terra è inquieta e inquietante. E tuttavia su questa isola-pianeta l'uomo, che ha qualcosa dell'ospite, vive una sua vita difficile, intensa, unica." (Manganelli, 2006, p. 83). Ed ancora: "La loro [degli islandesi] vita è in primo luogo la scienza del rapporto con l'incredibile terra in cui vivono." (Ibidem, p. 86). L'Islanda e gli islandesi, nella loro parabola storica ed evolutiva, rappresentano infatti un felice esempio di come l'uomo possa adattarsi a contesti ambientali apparentemente avversi traendovi non solo sostentamento ma anche prosperità, grazie ad un uso assennato ed una valorizzazione efficace delle risorse che potremmo definire "paesaggistiche" in senso lato. L'Islanda infatti, raggiunta la piena indipendenza nel 1944, ha registrato a partire dal secondo dopoguerra una dinamica di sviluppo tale da 
occupare oggi i primi posti nel mondo per ciò che riguarda il prodotto interno lordo pro-capite 2 e l'indice di sviluppo umano 3 , per citare solo gli indicatori più comuni. Alla base di tale sviluppo vi è un rapporto uomo-natura del tutto particolare, che informa le dinamiche insediative e socio-economiche, ma che al tempo stesso è profondamente radicato nella storia di questo territorio, tanto da configurarsi quale tratto culturale e identitario. L'Islanda ritrae le ragioni del proprio benessere principalmente dall'ambiente naturale: dal mare, che alimenta una fiorente industria della pesca, per tutelare la quale gli islandesi non esitarono a combattere le cod wars contro il Regno Unito e che rappresenta oggi la principale remora ad un ingresso nella Ue; dalla geotermia, che fa dell'Islanda il paese meno inquinato dell'Occidente con il più basso costo energetico pro-capite, oltre che un potenziale esportatore di energia elettrica; dal paesaggio naturale, che nella sua drammatica spettacolarità rappresenta una risorsa formidabile per un settore turistico in costante espansione. Riprendendo un tema già affrontato da Peris Persi ${ }^{4}$, si cercherà quindi di indagare il nesso ambiente-sviluppo che caratterizza il territorio islandese, focalizzando però l'attenzione sul ruolo del paesaggio naturale nella sfera culturale e nelle politiche di sviluppo del paese.

2. Il quadro paesaggistico - Il paesaggio quindi come risorsa da valorizzare, tema certo non nuovo né originale della ricerca geografica, che in Islanda assume tuttavia connotati peculiari e forse degni di approfondimento. Prima di entrare nel merito della questione, si vuole però affidare alle parole di alcuni acuti osservatori una descrizione - sia pur sintetica e immaginifica - del complesso quadro paesaggistico islandese, impresa peraltro non semplice, consideratene la varietà, l'eterogeneità, la stravaganza, la mutevolezza, la forza e la suggestività. Di certo l'Islanda sfugge a semplificazioni del tipo: terra di "ghiaccio e fuoco", di "cascate e geyser", di "fiordi e lagune”. L'Islanda è tutto questo, ma anche molto, molto di più. Scrive Valeria Viganò nel suo resoconto di viaggio: "L'Islanda non evoca solo letteratura, è un museo open air [...]. In questo paese non ci sono dipinti esposti all'interno o all'esterno di un museo, perché l'arte è la natura circostante. [...] In un caleidoscopico susseguirsi di vedute assolutamente reali, di proporzioni gigantesche se non fossero ridotte dalla nostra visuale umana, attraversiamo il più grande ma compatto e vario museo di pittura naturalistica e paesaggistica al mondo. Nell'arco di poche decine di chilometri vediamo marine, squarci collinari, vette innevate o fumanti, barche perse sull'orizzonte." (Viganò, 2004, p. 41). Ed effettivamente l'impressione che si ritrae da un breve viaggio in Islanda è innanzitutto quella di una straordinaria quanto repentina mutevolezza paesaggistica, di un'alternanza brusca ed imprevedibile di quadri ambientali unici, forti, lunari, di una bellezza quasi sconvolgente. Della qualità intrinsecamente tellurica e primigenia del paesaggio islandese, ai confini della indicibilità, ci dà invece efficacemente conto Manganelli: "In primo luogo, è la struttura iniziale, pre-umana, ai limiti del planetario, che offre quest'isola in cui non sembrano valere le regole di comportamento che hanno fatto della Terra un pianeta decorosamente stabile. Questa terra infantile ha tutte le bizzarrie, i furori afasici, i languori, le stravaganze oniriche di qualcosa che la Terra non ha ancora definitivamente accolto e domato. L'Islanda è una terra vulcanica, totalmente vulcanica, un continuo tremore, dovunque si spalancano forami che danno accessi a 
notturni fiumi ribollenti e provocano un'isterica ilarità i cartelli che dicono, in garbato inglese: non avvicinatevi, in caso di terremoto potreste cascarci dentro.” (Manganelli, 2006, p. 81). Segnaletica questa che ci dice qualcosa sull'attitudine islandese in materia di rischio ambientale: "con la natura non si scherza, ma senza isterismi!" A Manganelli fa eco Piero Citati, autore di un recente reportage per il quotidiano "La Repubblica": "Quando tornai a Roma, gli amici mi chiesero: «L'Islanda è bella? Ti sei divertito?» Ora, l'Islanda non appartiene alla categoria della bellezza: la bellezza è una cosa divina, o umana, o di una natura fatta a somiglianza dell'uomo e di Dio; mentre l'Islanda non conosce i nostri dèi, forse nessun dio, e non fa parte nemmeno, come diceva Manganelli, della natura. [...] All'inizio del ventunesimo secolo, uno vi trova le origini della terra, la fine dell'universo, le metamorfosi dei vulcani, gli incubi della natura e dell'uomo, l'Ade, gli abissi, i miracoli dell'acqua e del fuoco”. (Citati, 2004, p. 29). Dominano ancora i temi della "drammaticità" del paesaggio islandese e della sua "alterità" rispetto a ciò che è umano o comunque riferibile ad un universo familiare, conosciuto, sperimentato. L'Islanda e il suo paesaggio sembrano dunque sfuggire non solo alle definizioni stereotipe, ma anche agli schemi comuni di valutazione e di giudizio, che si tratti di estetica o geofisica poco importa. Altrettanto disumana appare Reykjavik - che, essendone la capitale, dell'Islanda riassume pregi e difetti - nel giudizio di Hlinur Biörn Hafsteinsson, abulico e indolente protagonista di 101 Reykjavík, il fortunato romanzo di Hallgrímur Helgason: "Raffiche di neve alla luce dei lampioni e atmosfera buia e mare gelido e salato... un intruglio... e spiagge cagliate. Poltiglia masticata intorno a penisole crude e fredde, montagne di antica formazione, rifiuti dimenticati, tumuli di cenere dell'era pagana, rottami dell'età del ferro. Escrementi glaciali induriti, ammuffiti e bianchi per la neve accumulata intorno a questa precaria città fatta di castelli di carte in cemento, un accampamento di costruzioni computerizzate destinate a sopravvivere per l'arco di una sola notte. [...] Nei cruscotti dei taxi caldi e nero-lucidi, in questi pezzi di plastica giapponese che veleggiano lenti per baie e fiordi coperti di ghiaccio, che vomitano i ritmi solari del pop californiano trasmesso dai programmi notturni dei canali radiofonici, proprio qui sta la conferma che questo paese è abitabile. Benessere e democrazia ben conservati nel vano portaoggetti." (Helgason, 2003, pp. 36-37). Ed ecco che dentro un paesaggio naturale che d'inverno può ben apparire inospitale, si colloca discreto un paesaggio umano fatto di legno e lamiere destinato a durare l'arco di una notte, per essere poi ricostruito e reinventato secondo i criteri funzionali della bioarchitettura ed i canoni estetici del design scandinavo.

3. L'etica ambientale in Islanda - Come è evidente, la relazione complessa e peculiare che la popolazione islandese intrattiene con il proprio contesto naturale è un aspetto centrale del nostro discorso sulla valorizzazione del paesaggio inteso come giacimento di risorse ambientali. Accanto a quella puramente economica, di cui si dirà in seguito, esiste tuttavia una dimensione etica ed in ultima analisi culturale, strettamente collegata alla prima in un rapporto di reciproca influenza, con risvolti che suscitano ammirazione ma anche - in qualche caso - perplessità.

Ad eccezione di alcune comunità perfettamente integrate e in sintonia con l'ambiente 
circostante, storicamente l'uomo ha guardato alla natura come a una inesauribile risorsa da sfruttare per il soddisfacimento dei propri bisogni, ignorando i principi della carriyng capacity. L'opinione pubblica assiste pertanto da lungo tempo, spesso impotente ma anche passiva, alla perdita di biodiversità, al depauperamento dei suoli, al dissesto dei corsi d'acqua, al surriscaldamento del pianeta e a molto altro di tal genere. La generale presa di coscienza della pericolosità dell'agire umano ha spinto l'uomo stesso a riflettere sul suo stile di vita ed ha di seguito portato al delinearsi di una nuova idea di natura. Quest'ultima infatti non venne più intesa esclusivamente come un bene-oggetto plasmato appositamente per il soddisfacimento dei bisogni e dei voleri dell'uomo-soggetto, ma anche come valore a sé stante e come soggetto etico. E' nata pertanto l'esigenza di estendere il campo di azione della filosofia e dell'etica alle istanze proprie della natura e ai rapporti che l'uomo, non più unico soggetto etico esistente, intrattiene con l'ambiente. Non è più sufficiente dunque la sola etica interpersonale, ma è necessaria anche un'etica ambientale: un pianeta in equilibrio è infatti condizione imprescindibile affinché vi sia un autentico sviluppo culturale, che ponga fine alla generale perdita dell'antica tradizione che lega ogni popolazione al proprio territorio.

Il senso di appartenenza è certamente uno degli aspetti più profondi del rapporto di rispetto, ma anche di intenso utilizzo, che lega i suoi pochi abitanti all'Islanda, divenuta in brevissimo tempo prospera e all'avanguardia sotto molti punti di vista, a partire da quello energetico.

L'isola islandese è geologicamente molto giovane e una costante metamorfosi sembrerebbe interessare il suo territorio, il quale potrebbe apparire come un campo di battaglia, dove i due indiscussi protagonisti dell'isola, i ghiacciai e i vulcani, si battono per la supremazia degli uni sugli altri. Non è però ancora stato scritto chi sarà il vincitore. Sono molti infatti i ghiacciai che hanno imprigionato all'interno del loro ventre uno o più vulcani, trionfando in tal modo su uno dei loro peggior nemici: la lava incandescente. Questa però solo in apparenza sembra aver perso la sua forza dirompente e il suo calore, poiché in realtà è sempre pronta ad ingoiare il ghiaccio sovrastante. Al di sopra delle eruzioni vulcaniche infatti il ghiacciaio crolla su sé stesso e l'antica lotta tra fuoco e ghiaccio si trasforma in una danza sinuosa, che si snoda a tratti lenta e a tratti velocissima, sprigionando calore e luce, intimorendo e affascinando, modificando quanto riesce anche solo a sfiorare sino a giungere all'oceano, il quale come un sipario nasconde e pone fine allo spettacolo.

Spettacolo cui gli islandesi non assistono in modo passivo e fatalista: la politica ambientale finora seguita dall'Islanda si ispira - con qualche importante eccezione di cui si dirà - alle istanze di rispetto e salvaguardia, poiché è proprio un ambiente in equilibrio all'origine dell'invidiabile crescita socio-economica, nonostante l'apparente severità di una natura "matrigna" che in effetti tale non è.

Come già sottolineato, Islanda non è sinonimo soltanto di ghiacciai, geyser e vulcani in eruzione; la sua realtà non si esaurisce in un tale insieme di opposti, che a volte si fondono, ma comprende contesti ambientali diversi, i quali in egual modo sono fonte 
di crescita e di sviluppo. All'origine della prosperità economica dell'Islanda vi sono l'oceano, l'energia geotermica e la straordinarietà di tutto il suo territorio, che continua a rivelarsi ai suoi abitanti ospitale e generoso, sorprendendo forse la restante parte del pianeta. L'Atlantico mitigato dalla Corrente del Golfo rappresenta oramai da secoli il principio e la ragione d'essere di numerose attività, che costituiscono sicuramente il pilastro portante dell'intera economia islandese e che trovano il loro comune denominatore proprio nel mare e in special modo nella pesca. A quest'ultima infatti sono strettamente legate numerose altre iniziative, sulle quali si fonda il benessere degli islandesi5.

Accanto alla pesca ed ai settori ad essa collegati, nuove modalità di rapportarsi all'ambiente vanno emergendo e sviluppandosi. Crescente è infatti il fascino che l'Islanda esercita su un sempre maggiore numero di visitatori (si veda par. successivo) attratti dalla verdeggiante e millenaria lava divenuta fertile, vivace e viva; dagli innumerevoli e variopinti torrenti glaciali, anch'essi ferventi di vita e fonte di nutrimento per migliaia di volatili; dagli antichi crateri trasformatisi oramai in brillanti laghi; dal fuggevole ma costante e tonante rumore-saluto dei geyser e in ultimo, ma non certo per l'intensità delle emozioni, dalla leggiadra danza delle balene. Queste ultime sono animali migratori che si spostano per riprodursi e nutrirsi; molte delle rotte seguite dalle balene si snodano lungo le coste ed è dunque possibile avvistarle ed osservarle da piccole imbarcazioni oppure dalla costa stessa. La straordinarietà insita nell'avvistamento e nell'avvicinamento a questi animali, il cosiddetto whale watching, rappresenta in Islanda uno dei pilastri del recente ecoturismo, il quale a sua volta costituisce un importante fonte di ricchezza che si va affiancando ai tradizionali canali economici.

Non v'è dubbio che la salvaguardia dell'ambiente e la consapevolezza della fragilità degli equilibri naturali abbiano accompagnato e profondamente influenzato sia lo stile di vita degli islandesi sia le decisioni socio-economiche dei diversi governi che si sono succeduti. L’intensa attività ittica, ad esempio, è stata regolata con lungimiranza e rispetto per la sua imprescindibile preservazione. Va tuttavia sottolineato che il peculiare rapporto che unisce gli islandesi al proprio territorio si fonda su un piano antropocentrico, in cui l'ambiente viene interpretato come una generosa cornucopia attraverso la quale soddisfare i bisogni dell'uomo. Ed è forse proprio su un presupposto di tal genere che poggia la recente decisione del governo islandese insieme a quelli di Norvegia e Giappone - di riprendere la caccia alle balene, nonostante la grande attrattiva che esse esercitano ed il danno di immagine che ne deriverà . Così come quella di sacrificare un'area di grande importanza naturalistica alla realizzazione di una diga destinata alla produzione di energia elettrica in grande quantità e a basso costo unicamente in funzione dell'istituendo sito industriale dell'Alcoa, multinazionale statunitense dell'alluminio. Sarebbe pertanto auspicabile che l'Islanda compisse un fondamentale, ma anche breve, passo verso un'etica ambientale a tutto campo, oltrepassando l'antropocentrismo e superando le contraddizioni che ancora caratterizzano la sua politica ambientale.

4. Paesaggio, sviluppo e "cultura" del paesaggio - "Il paesaggio si unifica attorno ad 
una tonalità affettiva dominante, perfettamente legittima malgrado sia refrattaria ad una riduzione puramente scientifica. Esso mette in causa la totalità dell'essere umano, i suoi legami esistenziali con la Terra, o, se si vuole, la sua geograficità originale: la Terra come luogo base e mezzo della sua realizzazione. Presenza lucida, che accomuna o estrania. E' la limpidezza di un rapporto che concerne la carne e il sangue." (Dardel, 1986, p. 34). Utilizziamo le parole di Eric Dardel per introdurre al nocciolo della questione: il territorio islandese, nella sua dimensione paesaggistica e naturalistica, come base e mezzo per la realizzazione di fortunate ed efficaci traiettorie di sviluppo di una comunità numericamente esigua, culturalmente omogenea, dai forti e condivisi caratteri identitari.

Ed ancora, con Dardel: "Si tratti di paura o di ammirazione o di simpatia, partecipiamo ancora, in quanto Moderni, tramite un accordo o una disarmonia fondamentali, al ritmo del mondo che ci circonda. Tra l'Uomo e la Terra si crea e si stabilizza una sorta di complicità nell'essere. Max Scheler lo ricordava: certi popoli hanno vissuto in uno "stato di fusione affettiva e vitale» con il mondo che noi chiamiamo «esterno»: ad esempio gli Indù." (Ibidem, pp. 14-15). Queste parole ben si attagliano, a parere di chi scrive, al caso islandese, dove "lo stato di fusione affettiva e vitale" con la natura si concretizza non tanto in una attitudine spirituale (che pure è presente e si manifesta in retaggi di credenze che attengono al mondo dell'animismo, del magico e dell'irrazionale, non del tutto scomparso dalla cultura immateriale - o forse dal subconscio - degli islandesiz), ma in un saggio misurarsi con le forze della natura e, laddove possibile, in un loro consapevole uso, che solo raramente sconfina nell'abuso. E' il caso della geotermia, che genera calore ed energia con un impatto ambientale quasi nullo ed a costi assolutamente competitivi (soprattutto se paragonati a quelli italiani). Ma anche e soprattutto della pesca, sottoposta al regime delle quote per mantenere intatte le capacità riproduttive dei banchi e per continuare a rappresentare la principale fonte di ricchezza del paese. Non è certamente questa la sede per approfondire le politiche ittiche ed energetiche, che dal secondo dopoguerra vanno caratterizzando le traiettorie di sviluppo dell'Islanda e che richiederebbero un lungo ed articolato discorso. Si vuole qui soltanto dire che, in ultima analisi, tanto lo spazio acqueo che circonda l'isola, quanto il "fumo bianchiccio [che esce dovunque] da meati terrestri" $\underline{8}$ (Manganelli, 2006, p. 53) e che segnala la presenza nel sottosuolo di potenziali risorse geotermali, possono ben essere considerati elementi tipici del paesaggio naturale islandese, nel senso indicato da Dardel. E non v'è dubbio che le attività legate alla pesca ed alla produzione di energia, sia essa di origine geotermica 0 idroelettrica, abbiano plasmato - e vadano ancora plasmando - il paesaggio "culturale" del paese.

Tuttavia, un nesso più diretto e immediato tra valorizzazione del paesaggio $\mathrm{e}$ traiettorie di sviluppo può essere individuato, come è evidente, nel turismo, che nello spettacolare paesaggio naturale islandese trova la sua principale ragion d'essere, come si diceva nel paragrafo precedente. Negli ultimi venti anni il settore è cresciuto, soprattutto grazie alla componente internazionale, ad un ritmo del 9\% annuo: basti pensare che dai 70 mila arrivi di turisti stranieri del 1982 si è passati ai 140 mila del 1992, per poi superare i 380 mila nel 2005. Oltre che del rapido trend di crescita, 
questi numeri danno conto della natura ancora elitaria e soft del turismo islandese, dai connotati quindi poco minacciosi per la capacità di carico del territorio così come per l'immagine di wilderness che l'isola evoca, da cui in buona sostanza traggono alimento i flussi summenzionati. La dotazione ricettiva dal canto suo va adeguandosi alla costante crescita della domanda, sia dal punto di vista quantitativo 9 che qualitativo, ed accanto a strutture tradizionali vanno moltiplicandosi in tutto il paese forme di accoglienza più moderne e adatte alle caratteristiche del territorio ed alla tipologia prevalente di turismo (vedi oltre), come $b \& b$ e guesthouses. I flussi stranieri provengono in massima parte - prevedibilmente, considerato il costo assai elevato di un soggiorno in Islanda - dai paesi scandinavi, seguiti da Regno Unito, Stati Uniti e Germania, anche se si registra un crescente numero di giapponesi, francesi e italiani. La stagionalità estiva è ancora forte, e si cerca di attenuarla attraverso politiche di prezzo che offrono pacchetti promozionali nei mesi invernali e nelle stagioni "morte". Un'analisi delle motivazioni effettuata su un campione di turisti negli anni 20012002 (Icelandic Tourist Board, 2004) conferma l'assunto iniziale di questa breve disamina: quasi l'8o\% degli intervistati ha scelto di effettuare un viaggio in Islanda nel periodo estivo perché attratto dal suo patrimonio naturale (in inverno, quando la natura è meno fruibile, la percentuale scende al 60\%). Le motivazioni strettamente culturali rappresentano all'incirca il 20\% delle risposte in estate contro il 30\% dell'inverno, mentre le forme improprie di turismo (d'affari, congressuale, familiare, di eventi, di studio e ricerca) variano dal 2-3\% al 10\% ciascuna 10 . Il notevole peso relativo del turismo si evince infine dai conti nazionali: questo settore partecipa per il $5 \%$ circa alla formazione del Pil e rappresenta, con il 13\%, la seconda fonte di valuta straniera per l'economia nazionale dopo l'export ittico.

Sempre in tema di valorizzazione economica del paesaggio, val la pena di menzionare la sagace politica varata dal governo agli inizi del XXI secolo per promuovere e attrarre in territorio islandese, in virtù delle sue speciali qualità paesaggistiche, investimenti nei settori cinematografico e televisivo attraverso il rimborso del $12 \%$ dei costi di produzione sostenuti in Islanda nel periodo 2001-2006. Il progetto, denominato Film-in-Iceland.org, ha suscitato un certo interesse a livello internazionale: accanto a pellicole islandesi cult come Næsland e Nói albínói praticamente sconosciute al di fuori della regione culturale scandinava - hanno beneficiato delle facilitazioni previste dal progetto film del livello di Batman Begins e Lara Croft: Tomb Raider, per citare solo le più note di una lunga serie di produzioni cinematografiche. Questa politica è interessante non tanto per le sue ricadute economiche - di certo non paragonabili a quelle garantite dal turismo - ma perché è indicativa di una "cultura" del paesaggio, di una consapevolezza che diventa fattore identitario e che permea molteplici aspetti della società islandese, da quello economico a quello politico, da quello ludico a quello artistico. Come suggerisce anche la grande considerazione di cui in Islanda gode la land art, singolare modo di coniugare arte e paesaggio: numerosi sono infatti gli artisti islandesi che si dedicano a questa particolare forma di espressione 1 , producendo "validi testimonials di un territorio che vuole farsi conoscere" (Borlenghi, 2004, p. 165). Ed una conoscenza del territorio viene veicolata anche attraverso i video-clip di musicisti islandesi di fama internazionale come Björk o i Sigur Rós, in cui spesso al paesaggio naturale 
dell'Islanda - location "georeferenziata" del filmato - viene attribuito un ruolo che non è di mera ambientazione, ma di amalgama espressivo e di identificazione emotiva con il contesto musicale.

5. Conclusioni - "Spoglia di cattedrali e pinacoteche, retta e sfidata da uomini pazienti e coraggiosi, l'Islanda ha il privilegio di poter dar piena aria a quella intima natura fantastica e magica, quel disvelamento delle forme naturali, e del nume che racchiudono, che non è più possibile in nessuna parte d'Europa." (Manganelli, 2006, p. 65). In queste poche parole viene sintetizzata, a parere di chi scrive, l'essenza della specificità islandese: contesto naturale unico, ingegno umano, memoria storica affidata a un patrimonio intangibile (data la carenza di quello tangibile), propensione verso le dimensioni immateriali dell'esistenza. Dall'interazione di questi fattori è scaturita la società islandese contemporanea, che se da un lato si serve di tecnologia all'avanguardia per soddisfare i propri bisogni materiali (e per rendersi la vita il più gradevole possibile), dall'altro fa a meno dei cognomi, chiama simi $\underline{12}$ il telefono e tölva 1.3 il computer, "crede" nelle fate e negli elfi. A far da sfondo, il paesaggio naturale: "ora capisco che, in Islanda, la forma naturale è comunicativa e significante per il suo modo di esistere, appartiene alla misteriosa araldica delle origini." (Ibidem, p. 63).

\section{Bibliografia}

Borlenghi E., "Un territorio alla ricerca di identità e la Land Art”, in Boll. Soc. Geogr. It., Roma, 2004, serie XII, vol. IX, pp. 163-168.

Brydon A., "Whale-Siting: Spatiality in Icelandic Nationalism”, in Pálsson G. e Durrenberger P. (cur.), Images of Contemporary Iceland. Everyday Lives and Global Contexts, Iowa City, University of Iowa Press, 1996, pp. 25-45.

Citati P., Viaggio in Islanda, Roma, La Repubblica, 23 agosto 2004.

Dardel E. L'Uomo e la Terra. Natura della realtà geografica (titolo orig.: L'Homme et laTerre. Nature de la réalité géographique), Milano, Unicopli, 1986.

Einarsson M., “The Wandering Semioticians: Tourism and the Image of Modern Iceland”, in Pálsson G. e Durrenberger P. (cur.), Images of Contemporary Iceland. Everyday Lives and Global Contexts, Iowa City, University of Iowa Press, 1996, pp. 215-235.

Einarsson N., "A Sea of Images: Fishers, Whalers, and Environmentalists”, in Pálsson G. e Durrenberger P. (cur.), Images of Contemporary Iceland. Everyday Lives and Global Contexts, Iowa City, University of Iowa Press, 1996, pp. 46-59.

Helgason H., 101 Reykjavík, Milano, TEA, 2003 (ed. orig. 1996). 
Icelandic Tourist Board, Tourism in figures, Reykjavik, 2004.

Manganelli G., L'Isola Pianeta e altri settentrioni, Milano, Adelphi, 2006.

Ministry for the Environment, Welfare for the Future. Iceland's National Strategy for Sustainable Development 2002-2020, Reykjavik, 2002.

Ministry for the Environment, Ministry of Fisheries, Ministry for Foreign Affairs, The Ocean. Iceland's Policy, Reykjavik, 2004.

Persi P., "L'Islanda: terra di calamità, terra promessa", in Orombelli G., Smiraglia C, Terranova R. (cur.), Verso una nuova geografia delle terre polari: sintesi e prospettive, Roma, Società Geografica Italiana, 1994, pp. 71-80.

Van Loon H. W., La geografia di Van Loon (titolo orig. Geography), Milano, Bompiani, 1949.

Viganò V., Siamo state a Kirkjubæjarklaustur, Vicenza, Neri Pozza Editore, 2004.

1 Luca Zarrilli ha redatto i parr. 1,2,4 e 5; Emanuela Finocchietti il par. 3.

$\underline{2}$ Fra i primi 10 paesi, con oltre 35mila \$ (ppp) al 2005.

$3 \mathrm{Al} 2^{\circ}$ posto dopo la Norvegia.

4 P. Persi ebbe a scrivere, condivisibilmente: "non esiste altro paese dove in simili condizioni si è sviluppata una comunità a così alto valore civile, a forte sviluppo tecnologico e ad elevato tenore di vita.” (Persi, 1994, p. 71).

$5 \mathrm{Al} 2002$ la pesca e la relativa industria di trasformazione rappresentavano complessivamente il 12,4\% del Pil, mentre i prodotti della pesca costituivano il 63\% delle esportazioni. Va tuttavia segnalato che il peso percentuale del settore va diminuendo nel tempo, se si considera che nel 1980 esso partecipava alla formazione del Pil per oltre il 15\% e garantiva circa il 75\% dell'export. E questo non già per un calo dei valori assoluti delle quantità di pescato e del relativo fatturato, ma per il ruolo crescente che vanno ricoprendo settori del terziario, soprattutto turismo e ICT.

$\underline{6}$ A tal riguardo, gli antropologi Anne Brydon e Níels Einarsson vedono nella decisione del governo islandese di riaprire la caccia alle balene - decisione peraltro sostenuta dalla maggioranza della popolazione - più che altro una volontà di affermazione della sovranità nazionale e del diritto di gestire l'ecosistema marino ricadente nella giurisdizione islandese in modo del tutto indipendente da interferenze 
esterne. Tale affermazione sarebbe inoltre supportata dal fatto che la caccia alle balene ha sempre contribuito in modo marginale all'economia nazionale e non rappresenterebbe un tratto culturale particolarmente sentito o rilevante per la popolazione islandese (Brydon, 1996, Einarsson N., 1996). Inoltre, il governo islandese sostiene da un lato la perfetta sostenibilità di una caccia contingentata e limitata a pochi esemplari, dall'altro la sua necessità al fine di preservare le capacità riproduttive dei banchi di pesca (Ministry for the Environment, 2002; Ministry for the Environment, Ministry of Fisheries, Ministry for Foreign Affairs, 2004).

Z Si veda al riguardo Manganelli, 2006, pp. 66-73.

$\underline{8}$ Non a caso Reykjavik significa "Baia dei Fumi”.

9 Da 244 esercizi e 12.471 letti del 2000 si è passati a 319 esercizi e 16.639 letti del 2005, con un incremento di oltre il 30\%.

$\underline{10}$ Gli intervistati potevano dare risposte multiple.

$\underline{11}$ Non è un caso che a Reykjavik si sia svolto nel giugno 2006 un interessante convegno internazionale dal titolo "SENSI/ABLE SPACES. Space, Art and the Environment", luogo di incontro tra geografia, antropologia ed arte. Nutrita la rappresentanza di artisti islandesi: Anna G. Torfadóttir vede nel potenziale espressivo offerto dalle fiabe popolari uno strumento di rivitalizzazione di paesaggi urbani in declino; Anna María Bogadóttir indaga sulle relazioni tra arte, tecnologia e vita quotidiana; Anna Snædís Sigmarsdóttir esprime nella sua arte la nostalgia per un perduto passato di armonia con la natura e una denuncia dell'attuale condizione di caos e disperazione; Guðrún Einarsdóttir si confronta con gli elementi basici della natura e con il passaggio e la manifestazione dell'energia; Osk Vilhjámsdóttir attraverso la sua opera svolge attività ambientalista militante; Steinunn porarinsdóttir svolge una riflessione sulla condizione umana nella società e nell'ambiente utilizzando sculture collocate nel paesaggio urbano di Reykjavik.

12 Dall'antico norreno sim, che significa cavo.

13 Letteralmente, "profetessa dei numeri”.

EMANUELA FINOCCHIETTI (b. 1975) holds a 2001 laurea degree in Foreign Languages and Literatures from the Università degli Studi di Viterbo "La Tuscia", her final dissertation dealing with Kurdish communities in former Soviet Union. In the same year she becomes adjunct by the Department of Human Sciences (DISU) of the same university, where she starts in 2005 her ongoing Ph.D. studies on the ethics of environmental protection and landscape valorisation as community heritage.

LUCA ZARRILLI (b. 1962) holds a 1988 laurea degree in Business and Economics from the Università degli Studi di Napoli "Federico II", his final dissertation being entitled "Strategies of intersectors diversification". In 1999 he completes his Ph.D. studies in Political Geography at the Università degli Studi di Trieste, with a thesis entitled "Albania. Geography of Transition". He has 
been Associate Professor in geographic disciplines at the Faculty of Economics of the Università degli Studi di Chieti-Pescara “G. D'Annunzio" since the year 2001, where he has taught the courses in Economic and Political Geography and, of late, Geography of Tourism, Geography of International Trade and Landscape and Environmental Geography. He has been extremely active in promoting, coordinating and participating in Erasmus/Socrates bilateral agreements between the Università degli Studi di Chieti-Pescara “G. D’Annunzio” and several European partners. 